\title{
PRIMERA FERIA GÉNERO Y SEXUALIDAD. SEMINARIO DE FORMACIÓN INTEGRAL FACULTAD DE HUMANIDADES Y CIENCIAS JURÍDICAS
}

Sandra Centeno Rojas*

\section{Introducción}

L a signatura de Seminario de Formación Integral contempla en uno de sus ejes el tema de Género. La educación sexual y reproductiva es uno de los temas que se incorporó con el propósito de contribuir en la descontrucción de patrones culturales que inciden en adolescentes y jóvenes, los cuales los hacen vulnerables a riesgos relacionados con la sexualidad y la reproducción.

Aunque se han hecho esfuerzos a nivel nacional para incidir en dicha problemática, seguimos enfrentando una pandemia hasta hoy en día. Jovencitas o mujeres enfrentan problemas como embarazos no deseados, abortos, violencia en las relaciones de género, enfermedades contagiosas VIH/ ITS.

La "Feria Educativa" fue el medio utilizado por las y los estudiantes, para convertirse en facilitadores de información relevante y pertinente sobre Género y Sexualidad. La misma incidió exitosamente en valores $\mathrm{y}$ actitudes de libertad, justicia, igualdad, tolerancia, relaciones de equidad de género, respeto a la diversidad sexual y compromiso social de estudiantes de las carreras Trabajo Social, Derecho, Diplomacia y Ciencias Políticas, Filología y Comunicación. Pero sobre todo en los prejuicios y concepciones que los jóvenes y adultos tienen sobre la sexualidad.

Los jóvenes recopilaron y expusieron la información relevante y pertinente relacionada con los siguientes temas: mitos y realidades en las relaciones sexuales, la sexualidad sin exclusión enfocado en las personas con discapacidad, métodos de planificación en mujeres y hombres, la procreación responsable y el uso de anticonceptivos, tipos de disfunciones sexuales en las mujeres y hombres, la penalización del aborto terapéutico y sus efectos, enfermedades de transmisión sexual, Ley 779, entre otros.

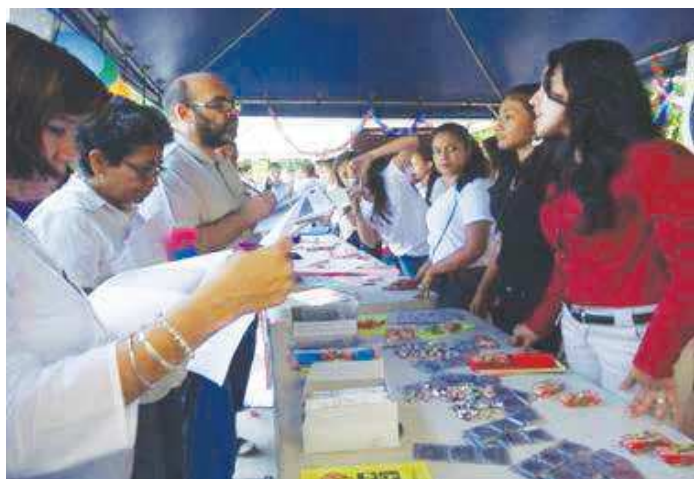

Los jóvenes recopilaron y expusieron la información relevante y pertinente relacionada con los siguientes temas: Mitos y Realidades en las relaciones sexuales, la sexualidad sin exclusión enfocado en las personas con discapacidad, métodos de planificación en mujeres y hombres, la procreación responsable y el uso de anticonceptivos, tipos de disfunciones sexuales en las mujeres y hombres, la penalización del aborto terapéutico y sus efectos, enfermedades de Transmisión Sexual, La Ley 779, entre otros.

* Msc en Historia y docente del Departamento de Historia UNAN-Managua. 
El proceso de consulta y estudio facilitó a las y los estudiantes la elaboración de proyectos creativos en plegables, pósteres, murales, foto-reportajes, entre otros. De esta manera se puso en práctica el aprendizaje sustentado en la experiencia, pues se aprende al hacer y al reflexionar sobre lo que se hace.

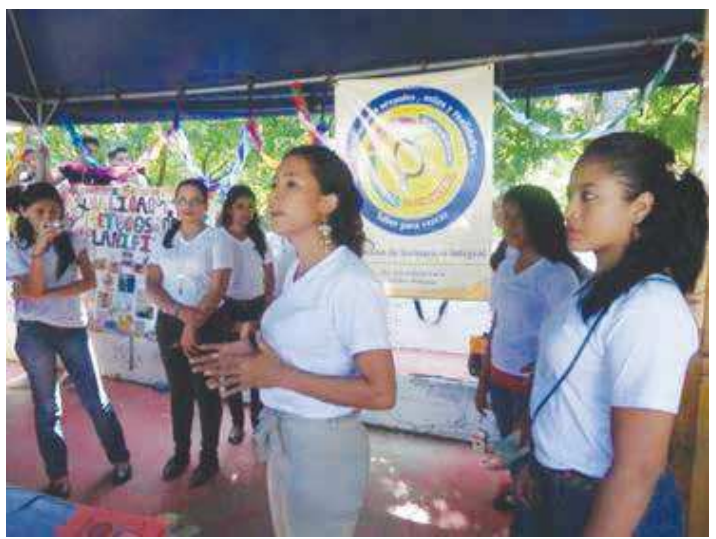

El proceso de consulta y estudio facilitó a las y los estudiantes la elaboración de proyectos creativos en plegables, pósteres, murales, foto-reportajes, entre otros. De esta manera se puso en práctica el aprendizaje eminentemente experiencial, pues se aprende al hacer y al reflexionar sobre lo que se hace

La dinámica del desarrollo de la feria se concentró en la exposición de los conocimientos adquiridos. Su culminación se dio con la evaluación y premiación de los mejores proyectos creativos cuyo escrutinio estuvo a cargo de un Jurado integrado por docentes que imparten la asignatura de Seminario de Formación Integral.

Esta primera experiencia motiva seguir aportando en esta temática, no sólo por los beneficios que conlleva esta actividad a la comunidad educativa del Alma Mater, sino también a la sociedad en su conjunto. La educación sexual libre de prejuicios culturales, es una demanda social hoy en día. De tal manera que la Universidad debe seguir contribuyendo en la educación de las nuevas generaciones.

\section{La educación Sexual sin prejuicios culturales}

La educación como una Institución social, históricamente ha reproducido valores patriarcales. De igual manera otras instituciones como la familia y la iglesia han sido en el proceso de socialización las responsables de educar y reproducir valores que han incidido en las desigualdades sociales de género.

Las características fisiológicas y sexuales (biológicas) propias con las que nacemos son naturales y no se modifican, pero somos diferentes porque en cada cultura, en cada sociedad, el significado de ser mujer o de ser hombre cambia según normas, valores, ideas, concepciones, puntos de vista. Las prácticas sexuales también son parte de una construcción social y cultural. Estas se viven e interpretan de forma diferente de acuerdo al lugar donde nos encontremos.

La sexualidad debe entenderse como un componente más de la personalidad de los seres humanos. Es una fuente de comunicación, afecto, placer, expresión del deseo y reproducción. Razón por la cual la sexualidad no hace referencia únicamente a las partes genitales o aparato reproductivo del cuerpo, sino también tiene que ver con las relaciones establecidas con otras personas y con nosotras/os mismas/ os, la comunicación, los sentimientos, las emociones, el placer y el cariño, la felicidad, el sentirse bien, en fin con todas las expresiones humanas (Guzmán 2012, pág. 21)

La educación sexual debe contribuir en la convivencia y la armonía sexual entre hombres y mujeres. En el derecho a ejercer una sexualidad y reproducción con libertad. Debe de permitir al ser humano el disfrute de una sexualidad sana, responsable, segura y con el menor riesgo posible. 
Con la participación de las y los estudiantes en esta feria se incidió de forma significativa en la toma de conciencia, en la apropiación del conocimiento sin temor a los prejuicios. De igual manera se constató como algunas jovencitas con naturalidad, se referían a la utilización de los métodos anticonceptivos como el condón.

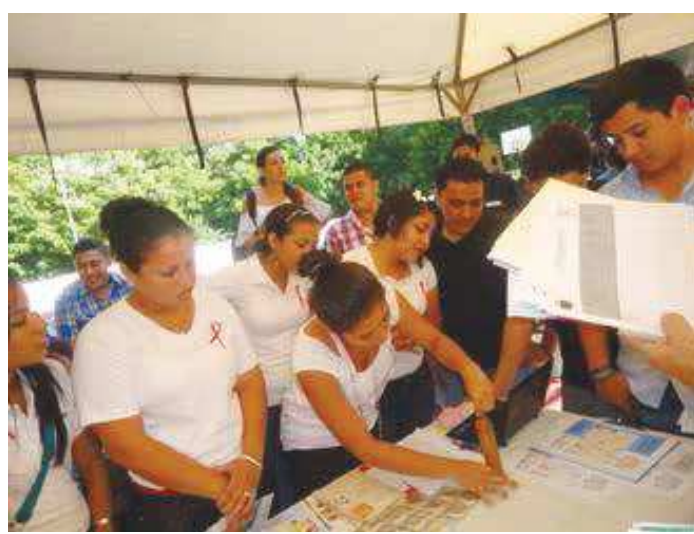

Con la participación de las y los estudiantes en esta feria se incidió de forma significativa en la toma de conciencia. Esto se pudo demostrar en la apropiación del conocimiento sin temor a los

prejuicios. Se pudo demostrar como algunas jovencitas se referían a la uti lización de los métodos anticonceptivos como es el caso del condón con naturalidad.

Para Paulo Freire, educar es poder transformar la realidad circundante como un medio esencial para crear una sociedad a base de su propia cultura. Se pretende que la educación pueda dar lugar a una conciencia pura y natural, de la justicia como fundamento la "Liberación". No debe ser impuesta sino por el contrario debe ser el trabajo de cada una de las personas que cohabitan un determinado espacio que se conoce como país, donde existen personas capaces y competentes $(2004,45)$.

Los derechos humanos son partes inherentes de la sexualidad. Hombres y mujeres deben de gozar de los derechos implícitamente definidos en el ejercicio de la sexualidad y la reproducción. Por lo que el reconocimiento pleno de este derecho nos permite tomar libremente decisiones sobre nuestro cuerpo de manera autónoma,

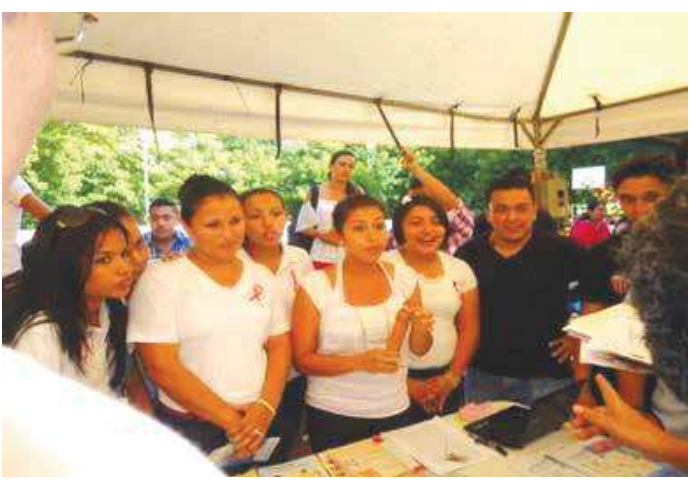

Para Paulo Freire, educar es poder transformar la realidad circundante como un medio esencial para crear una sociedad a base de su propia cultura, se pretende que se comprenda que a través de la educación se puede dar lugar a una conciencia pura y natural de la justicia que tiene como fundamento la "Liberación", que no debe ser impuesta sino por el contrario debe ser el trabajo de cada una de las personas que cohabitan un determinado espacio que se conoce como país donde existen personas capaces y competentes $(2004,45)(2004,45)(2004,45)(2004,45)$ $(2004,45)(2004,45)$.

con independencia de la edad, sexo, raza, condición social, religión, etc sin que esto genere discriminación o violencia. (Op.cit 2012, pág. 11)

\section{Conclusiones}

La juventud demostró interés por el aprendizaje y creatividad en la elaboración de proyectos creativos. Se pudo constatar además que en el desarrollo de la feria se compartió experiencias individuales y colectivas referidas a la temática de la sexualidad.

El conocimiento científico adquirido sobre cada tema refuerza el conocimiento empírico de la juventud. Es el eslabón más significativo para comprender $\mathrm{y}$ tomar conciencia de lo que significa la sexualidad.

La educación sexual es una necesidad y en este sentido la Universidad debe seguir aportando en la construcción de nuevos paradigmas que contribuyan a ejercer una sexualidad sana, segura, placentera $\mathrm{y}$ sin prejuicios culturales en la juventud nicaragüense. 


\section{Referencias Bibliográficas}

Centeno Sánchez, Mauricio. Proyecto primera feria educativa: género y sexualidad. Managua 11 de septiembre de 2013

Freire, Paulo. Pedagogía de la Autonomía. Editorial Paz y Tierra,S.A. Sao Paulo, Brasil, 2004.

Guzmán Villagra, Marcelino. Hablemos de sexo y otras cosas. Managua, Nicaragua Ipas, Centroamérica, 2012.

\section{Anexos}

\section{Algunos Proyectos creativos presentados}
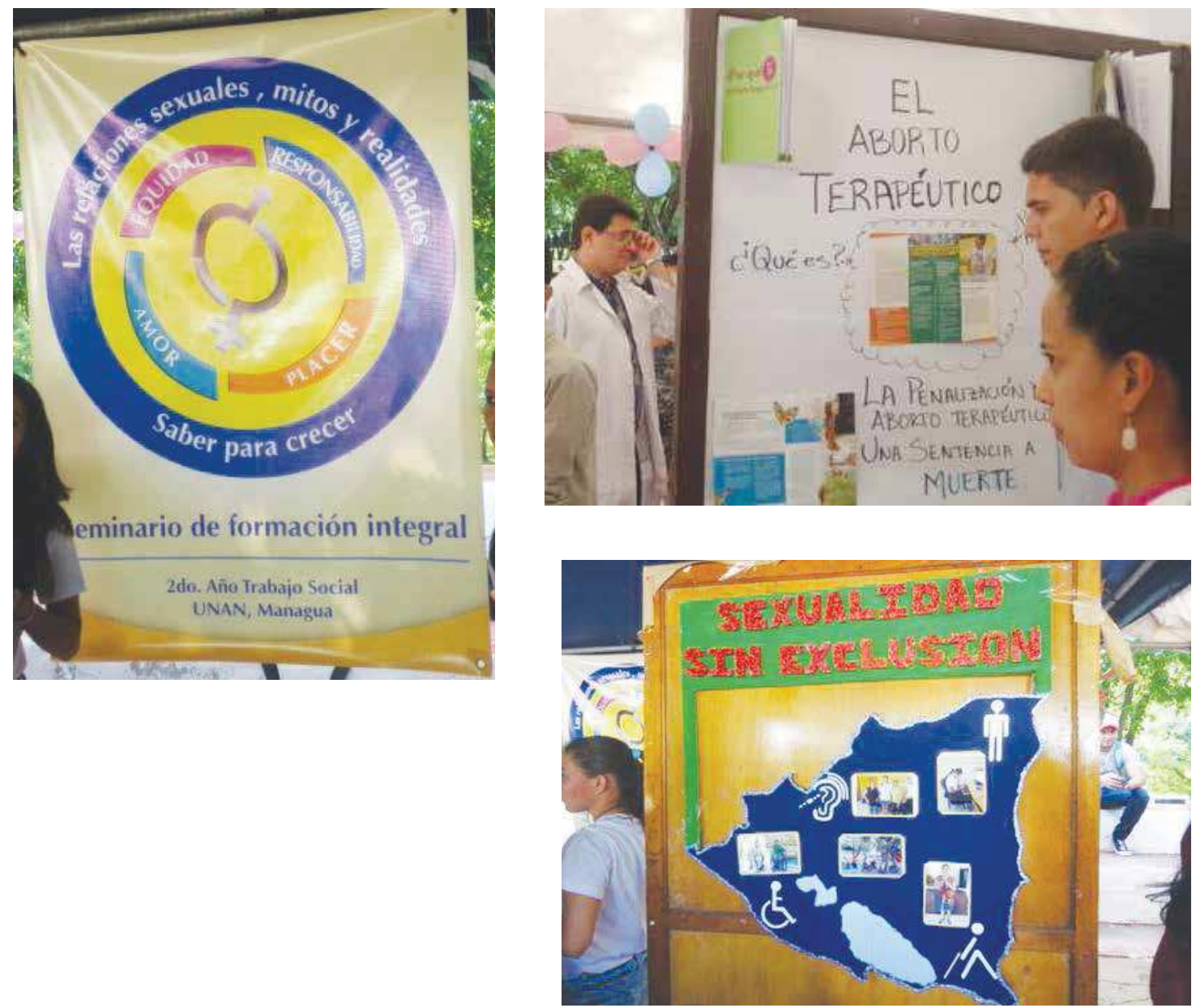


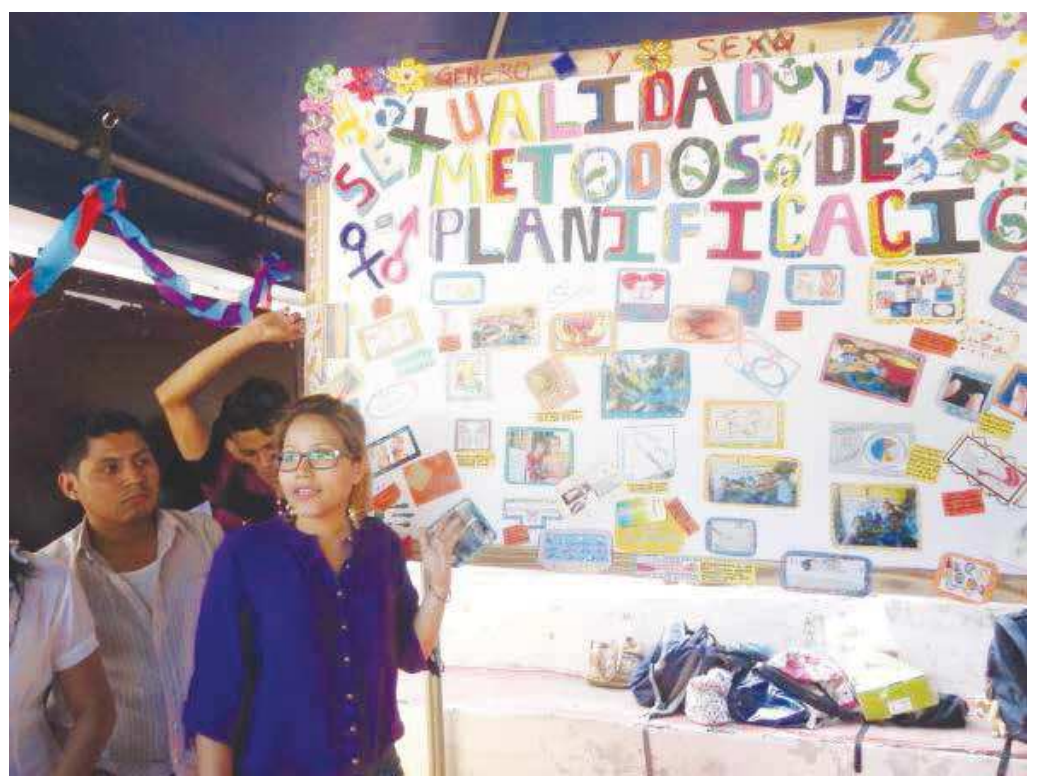

\section{Jurado que evaluó los proyectos creativos}

Luis Alfredo Lobato Blanco Decano de la Facultad de Humanidades y Ciencias Jurídicas Teresa Cabrera Coordinadora de la asignatura de Seminario de Formación Integral (SFI)

Facultad de Ciencias.

Elida Solórzano Coordinadora de la asignatura de Seminario de Formación Integral (SFI) Facultad de Educación e Idiomas
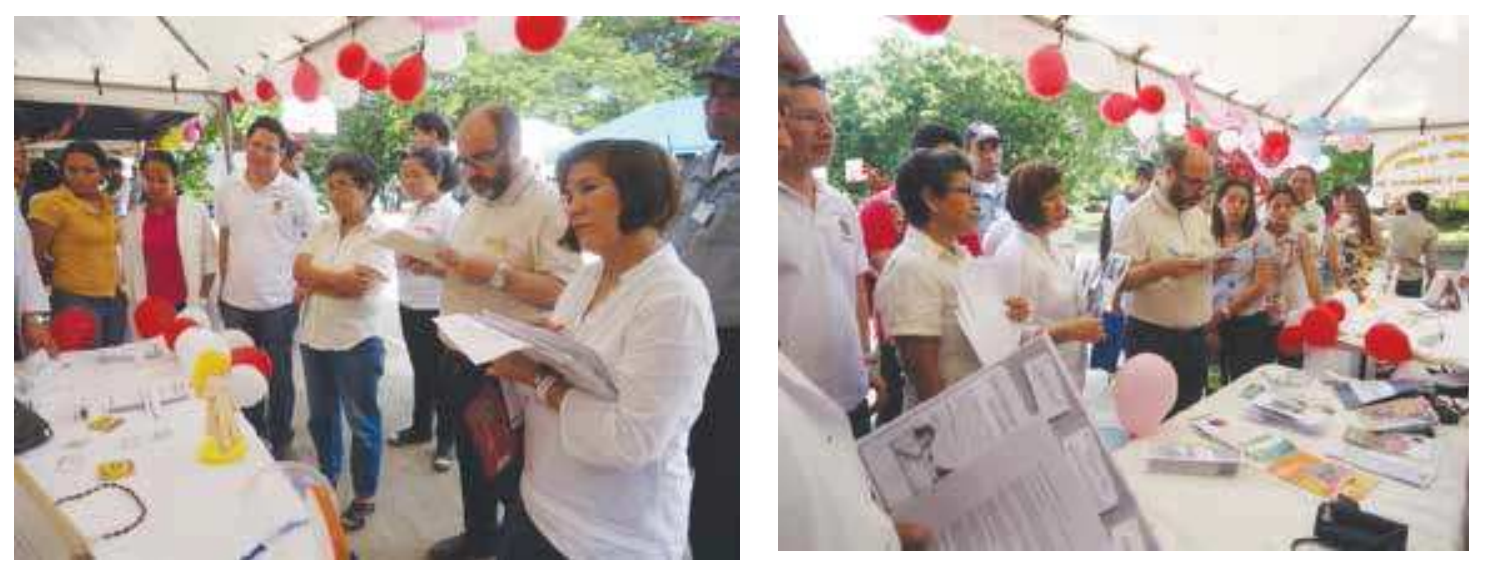

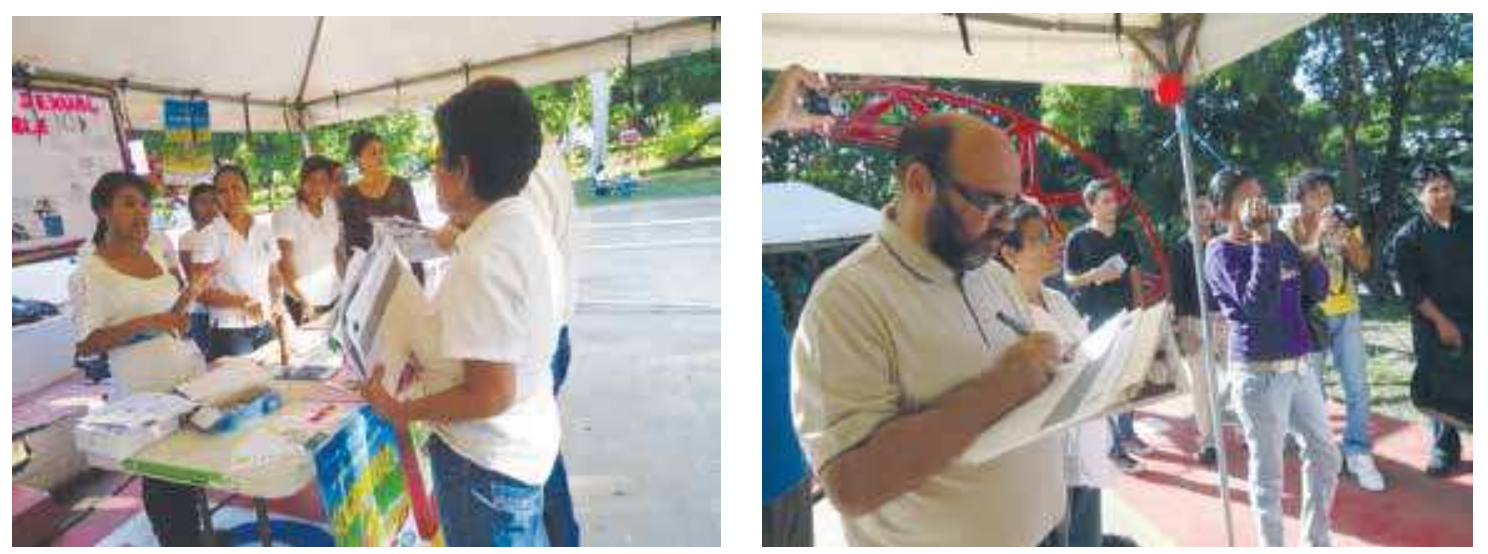

Entrega de Diplomas a proyecto Ganador

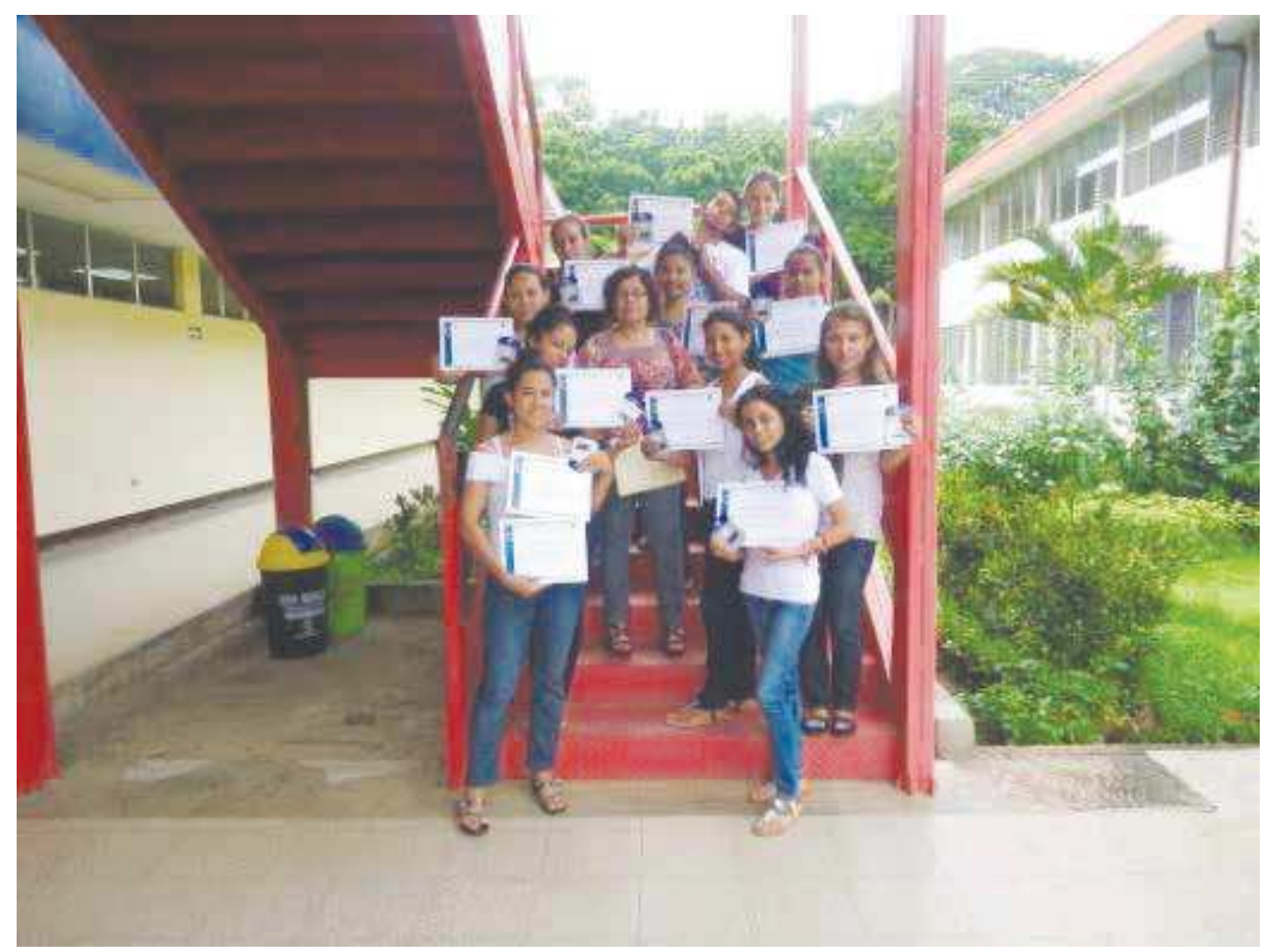

Proyecto "Saber para crecer: Sexualidad, mitos y realidades" Estudiantes que ganaron el primer lugar Carrera Trabajo Social

Sandra Centeno Rojas Profesora y Coordinadora de la asignatura SFI 